Proc. of the 15th Int. Workshop on Slow Positron Beam Techniques and Applications, Prague, September 2-6, 2019

\title{
On the Characterization of Small-Scale Samples Using Radioisotope Positron Sources
}

\author{
M. Saro*, V. Krsjak, M. Petriska and V. Slugen \\ Institute of Nuclear and Physical Engineering, Faculty of Electrical Engineering and Information Technology, \\ Slovak University of Technology, Ilkovicova 3, Bratislava, 812 19, Slovakia

\begin{abstract}
Probing of small-scale samples such as thin ion-implanted layers or TEM discs using conventional radioisotope sources is a rather complicated task due to the range of energies of the emitted positron. Besides, experimental spectra obtained from samples containing internal transmutation-based positron source are naturally disturbed by positron annihilation outside of the sample. To estimate these contributions to the spectra, the Geant4 simulation toolkit was used. In addition to various absorption profiles of positrons from realistic encapsulated sources, the use of TEM disc containing ${ }^{44} \mathrm{Ti} /{ }^{44} \mathrm{Sc}$ as an external positron source is discussed and suggested.
\end{abstract}

DOI: 10.12693/APhysPolA.137.193

PACS/topics: positron annihilation, radioisotope source, depth analysis

\section{Introduction}

The evaluation of radiation damage plays an important role in the development of new materials for nuclear applications. The microstructural characterization is required to understand the mechanism behind the changes in mechanical properties. Ion implantation appears to be a suitable way of studying radiation effects in materials. In the majority of the cases, however, it leads to the near-surface material damage. A powerful tool for inspection of defects created by ion implantation is the variable energy positron annihilation spectroscopy (VEPAS). The depth of inspection can be chosen by adjusting the kinetic energy of monochromatic positron beams from a few $\mathrm{nm}$ to several $\mu \mathrm{m}$, depending on the inspected material [1]. In the first approach, the positron energy spectrum from radioisotope sources such as ${ }^{22} \mathrm{Na}$ or ${ }^{44} \mathrm{Ti} /{ }^{44} \mathrm{Sc} \mathrm{[2]} \mathrm{consists} \mathrm{of} \mathrm{energetic} \mathrm{positrons} \mathrm{which} \mathrm{pen-}$ etrate material up to several tens of $\mu \mathrm{m}$. Nevertheless, a certain amount of the positrons annihilate in the nearsurface area as well. Naturally, the geometry of the measurement has a significant influence on the energy spectrum of implanted positrons too [3, 4], therefore, some shifts in the spectrum can be observed. For the quantitative analysis, we have carried out a series of simulations to give an answer on the feasibility of observation of the near-surface regions with a radioisotope positron source.

\section{Measurement and simulation model}

Geant4 is a suitable simulation toolkit used for the evaluation of radioisotope contribution to thin layer inspected areas. Positron, unlike electron, mostly loses its kinetic energy via collisional energy losses rather than

*corresponding author; e-mail: matus.saro@stuba.sk radiative ones. So, the main energy losses interactions of positrons with matter are inelastic collisions, where secondary particles are ejected such as electrons or gamma and ions are formed. Similarly, to [5] we chose our default physics list to be electromagnetic PENELOPE (PENetration and Energy LOss of Positrons and Electrons) model. For purposes of source decay behavior, we implemented radioactive decay physics list. Each simulation event begins with the creation of ${ }^{22} \mathrm{Na}$ or ${ }^{44} \mathrm{Ti}$ ion, respectively, with zero kinetic energy and no excitation energy, randomly within the source volume. In this particular case, we omit to track any other secondary particles other than positrons (electrons, ions, antineutrons, and gamma). The initial energy of positron is primarily collected and this positron is then tracked until it leaves Kapton foil or TEM disc to the side of the sample, where it annihilates.

We have performed simulations from two different sources. In the first case of ${ }^{22} \mathrm{Na}$ source, the geometry of simulation consists of the $\mathrm{NaCl}$ compound, which is in the center of geometry. The source was modeled as a $1 \mu \mathrm{m}$ thin cylinder with a diameter of $1.5 \mathrm{~mm}$. It is surrounded by two Kapton foils, one each side with a thickness of $7.5 \mu \mathrm{m}$ and square dimensions of $1.5 \times 1.5 \mathrm{~cm}^{2}$. The gap between the two foils is filled with adhesive glue material which we chose to be $\mathrm{SiO}_{2}$. With various materials, classic sandwich geometry was simulated, with sample dimension $1 \times 1 \mathrm{~cm}^{2}$ and variable thickness.

The second simulation was performed using the ${ }^{44} \mathrm{Ti} /{ }^{44} \mathrm{Sc}$ source. The geometry of this source and the chemical composition was selected to reflect a typical TEM disc sample, used in various irradiation experiments, with a diameter of $3 \mathrm{~mm}$ and thickness of $250 \mu \mathrm{m}$. Due to the availability of several TEM samples of ferritic/martensitic steels, irradiated in the spallation source, we have used the chemical composition of Fe9Cr1W alloy. Titanium is evenly distributed within the TEM sample. The sample geometry remained the same as in the previous case. 


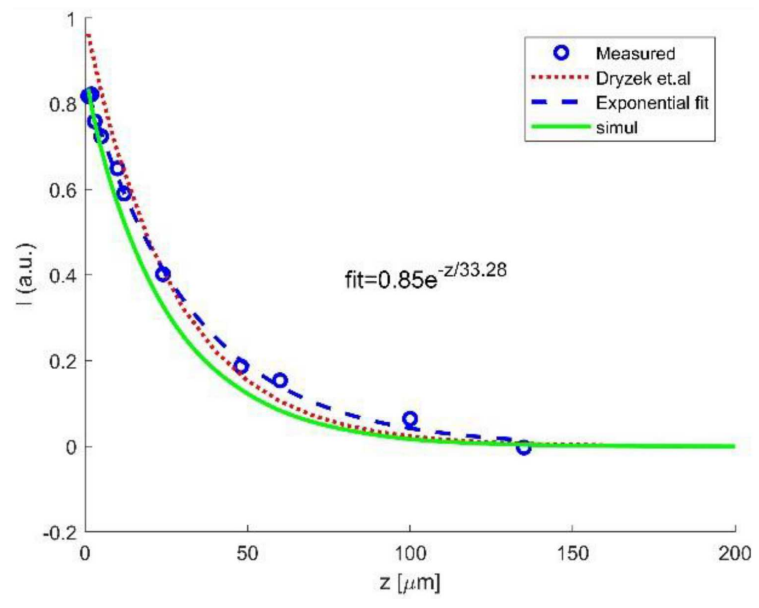

Fig. 1. Stopping profile of positrons from Kapton $(7.5 \mathrm{~mm})$-encapsulated ${ }^{22} \mathrm{Na}$ source in Fe obtained from the measurements on thin $\mathrm{Fe}$ and $\mathrm{FeCr}$ foils together with Geant4 simulation stopping profile in $\mathrm{Fe}$.

For purposes of simulation model validation, series of thin Fe and FeCr foils measurements with UPILEX backing have been carried out from thickness of $1 \mu \mathrm{m}$ to $135 \mu \mathrm{m}[6]$. As can be seen in Fig. 1a reasonable agreement has been achieved between the measurement results and the simulation, so the model could be considered as validated.

\section{Results and discussion}

The obtained results depicted in Figs. 2 and 3 show that the conventional Kapton encapsulated ${ }^{22} \mathrm{Na}$ source provides the most information from volumes between the thickness of $7 \mu \mathrm{m}$ to $50 \mu \mathrm{m}$ when Fe material is inspected. This corresponds in the ferrous sample to a volume modified by helium implantation with energy ranging from $3.5 \mathrm{MeV}$ to $14 \mathrm{MeV}$. For near-surface regions between $1 \mu \mathrm{m}$ to $3 \mu \mathrm{m}$, the positron annihilation ratio is rather small and the contribution of this volume to the final spectrum equals or is even below the contributions from outside of the inspected volume (Kapton, $\mathrm{NaCl}$, air, holders, etc.).

The limit of $10 \%$ of annihilated positrons can be reached at a thickness of $3 \mu \mathrm{m}$. The most significant rise of the positron annihilation contribution in a sample is between the thickness of $7 \mu \mathrm{m}$ to $45 \mu \mathrm{m}$. From this point, positron annihilation percentage in sample slowly rises from $65 \%$ to a maximum of $80 \%$ of all annihilated positrons, which happens at a thickness of $120 \mu \mathrm{m}$. A contribution of $20 \%$ to the final annihilation spectrum remains within the encapsulated source. Inspection of near-surface volumes with ${ }^{44} \mathrm{Ti} /{ }^{44} \mathrm{Sc}$ source without positron thermalization is not viable because of high positron kinetic energy. Positrons from ${ }^{44} \mathrm{Ti} /{ }^{44} \mathrm{Sc}$ source penetrate up to $600 \mu \mathrm{m}$ in ferrous materials. With TEM disc described in Sect. 2, penetetration depth is only $450 \mu \mathrm{m}$. In following results, limits
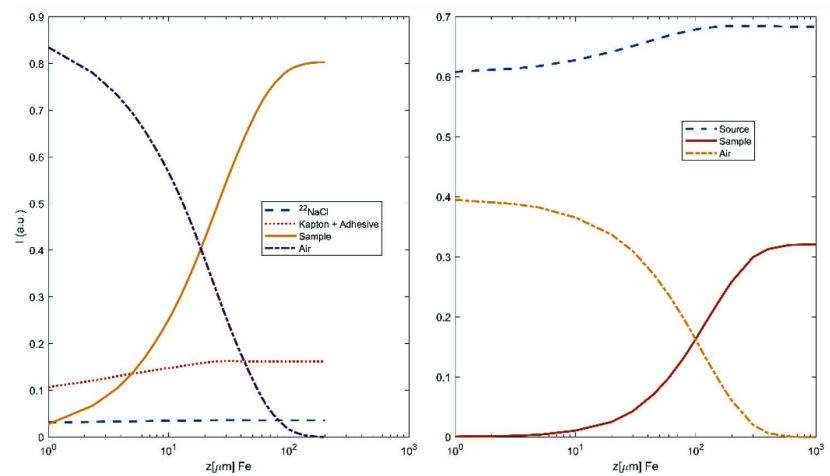

Fig. 2. The ratio of positron annihilation sites from Geant4 simulation with varying thickness of Fe sample, with encapsulated sodium source (left) and with TEM disc-shaped ${ }^{44} \mathrm{Ti} /{ }^{44} \mathrm{Sc}$ source (right).

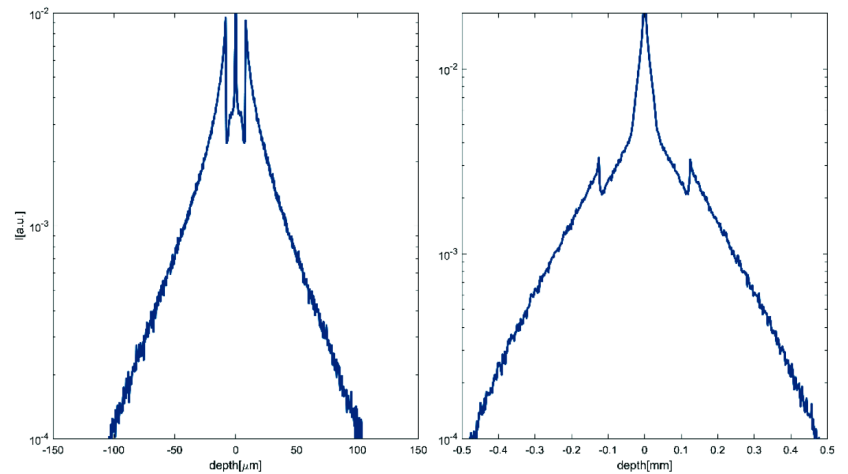

Fig. 3. Positron stopping profile with $1 \mu \mathrm{m}$ thick ${ }^{22} \mathrm{Na}$ source, $7.5 \mu \mathrm{m}$ Kapton foil and $1 \mathrm{~mm}$ thick Fe samples in sandwich geometry (left) and positron annihilation profile with $250 \mu \mathrm{m}$ thick ${ }^{44} \mathrm{Ti} /{ }^{44} \mathrm{Sc}$ source and $1 \mathrm{~mm}$ thick Fe samples in sandwich geometry (right).

for mentioned TEM disc will be presented, although results for $5 \mu \mathrm{m}$ thin foil ${ }^{44} \mathrm{Ti} /{ }^{44} \mathrm{Sc}$ source vary from these values and will be presented in brackets, to show possibilities of ${ }^{44} \mathrm{Ti} /{ }^{44} \mathrm{Sc}$ source in conventional PAS. $10 \%$ of annihilated maximum positrons in sample occur in the first $60(27) \mu \mathrm{m}$. The region of the positron stopping profile lies between $60(27) \mu \mathrm{m}$ and $300 \mu \mathrm{m}$. In the case of using TEM disc containing ${ }^{44} \mathrm{Ti} /{ }^{44} \mathrm{Sc}$ as an external positron source, 68 (23)\% of positrons annihilate within the disc. This contribution remains the same, if the ferrous sample has a thickness higher than $120 \mu \mathrm{m}$.

\section{Conclusions}

From the presented work we can conclude, that encapsulated unmoderated ${ }^{22} \mathrm{Na}$ source can be used for the PAS characterization of volumes extending more than $3 \mu \mathrm{m}$ below the surface. In the case of irradiation studies with specific damage profiles (ion implantations), displacement damage values must be weighted 
over the positron implantation profile. Unmoderated $5 \mu \mathrm{m}$ foil ${ }^{44} \mathrm{Ti} /{ }^{44} \mathrm{Sc}$ source is useful for bulk studies rather than near-surface region when sandwich measurement geometry is used and Fe based materials are inspected. In these measurements, source contribution can achieve up to $23 \%$. TEM disc usage as a positron source is also possible within ranges presented in results, but detailed characterization of source components is needed before any application in PAS.

\section{Acknowledgments}

This work was financially supported by Scientific Grant Agency of the Ministry of Education of Slovak Republic and the Slovak Academy of Sciences No. VEGA 1/0104/17.

\section{References}

[1] C. Hugenschmidt, Surf. Sci. Rep. 71, 547 (2016).

[2] V. Krsjak, Y. Dai, J. Nucl. Mater. 465, 311 (2015).

[3] M. Saro, V. Krsjak, M. Petriska, V. Slugen, AIP Conf. Proc. 2131, 020039 (2019).

[4] X. Ning, X. Cao, C. Li, D. Li, P. Zhang, Y. Gong, R. Xia, B. Wang, L. Wei,Nucl. Instrum. Methods Phys. Res. B 397, 75 (2017).

[5] L.Y. Dubov, Y.A. Akmalova, S.V. Stepanov, Y.V. Funtikov, Y.V. Shtotsky, Acta Phys. Pol. A 132, 1482 (2017).

[6] M. Saro, V. Krsjak, M. Petriska, V. Slugen, AIP Conf. Proc. 2182, 050016 (2019). 